\title{
NEMO : on t'a dans la peau !
}

Le facteur de transcription NF- $\kappa \mathrm{B}$, découvert il y a 15 ans dans le laboratoire de D. Baltimore, apparaît jouer un rôle fondamental dans de nombreux processus physiologiques comme la réponse immunitaire et inflammatoire, l'adhérence cellulaire, l'oncogenèse et l'apoptose. Localisé dans le cytoplasme où il est associé à la molécule inhibitrice IKB, il migre dans le noyau en réponse à une multitude de stimulus et règle l'expression de nombreux gènes. Son mode d'activation particulier a fait l'objet d'intenses recherches durant les 7-8 dernières années. Il peut être divisé grossièrement en trois phases. $\mathrm{Au}$ cours de la première, IKK (IкB kinase), un complexe multiprotéique de haut poids moléculaire (700900 kDa) est activé. Dans un second temps, IKK phosphoryle deux résidus sérine spécifiques sur I $\mathrm{KB}$, provoquant son ubiquitination puis sa dégradation par le protéasome, ce qui permet la libération de NF- $\kappa \mathrm{B}$ qui rejoint le noyau où il active ses gènes cibles ([1] et $m / s$ 1999, $n^{\circ} 3$, p. 419).

IKK est constitué d'au moins trois sous-unités distinctes. Deux ont une activité catalytique, IKK- $1 / \alpha$ et IKK$2 / \beta$ [2] alors que la troisième, NEMO (pour $N F-\kappa B$ essential modulator, ou IKK- $\gamma /$ Fip-3/IKKAP), participe à leur activation et régulation. L'implication de NEMO dans l'activation de $\mathrm{NF}-\kappa \mathrm{B}$ fut démontrée lors de son clonage, reposant sur la complémentation génétique d'une lignée fibroblastique mutante ayant perdu la capacité d'activer NF- $\kappa \mathrm{B}$ en réponse à une large variété de stimulus [3].

Si le rôle clé de NF-kB dans le système immunitaire fut établi très tôt après sa découverte, ce n'est que beaucoup plus récemment que sa fonction au niveau de l'épiderme a été révélée. En utilisant des souris transgéniques surexprimant NF- $\mathrm{KB}$ ou en inhibant son activation, et cela spécifiquement dans le compartiment cutané, le groupe de P. Khavari a démontré que ce facteur de transcription joue un rôle double au niveau de l'épiderme: d'une part, il protège les kératinocytes de l'apoptose durant leur différenciation et d'autre part il contrôle leur prolifération [4].

La participation de NF-кB dans le compartiment cutané a également été observée chez des souris invalidées pour chacun des gènes codant pour les deux sous-unités catalytiques de IKK. Le phénotype de ces souris est très différent. Celui des souris $I K K-2^{-/-}$était prévisible, semblable à celui des souris invalidées pour le gène relA, qui code pour une des sous-unités de NF-אB: les embryons meurent en effet vers le jour 12,514,5 du développement d'une apoptose hépatique massive, et l'on observe une absence d'activation de NF- $\kappa \mathrm{B}$ en réponse aux cytokines [5]. En revanche, les souris $I K K-1^{-/-}$survivent jusqu'à la naissance, mais présentent un aspect très caractéristique, avec leurs membres engoncés dans un épiderme épais. Une analyse plus fine indique que, chez ces souris, les kératinocytes prolifèrent de façon intense et ne se différencient pas. Cela suggère que la sous-unité IKK-1 n'est pas indispensable à l'activation de NF-kB en réponse aux cytokines mais participe, d'une façon qui reste à définir, au développement des kératinocytes et de la peau [6].

La démonstration la plus spectaculaire du rôle majeur que joue le facteur de transcription NF- $\kappa \mathrm{B}$ dans l'épiderme vient en fait d'être récemment apportée grâce à l'identification du gène responsable de la génodermatose humaine incontinentia pigmenti (IP) [7]. Cette maladie liée à l'X, létale très tôt au cours du développement chez le garçon, se caractérise chez la femme par une patholo- gie complexe au niveau de l'épiderme. Peu de temps après leur naissance, les petites filles développent une dermatose qui évolue en suivant 4 stades distincts. Dans un premier temps, au cours des deux premières semaines de la vie, apparaissent des lésions vésiculo-bulleuses assez semblables à celles qui caractérisent l'éruption associée à la varicelle. Puis, entre la deuxième et la sixième semaine, des lésions verruqueuses surviennent, précédant un stade d'hyperpigmentation cutanée dû à une accumulation de mélanine. Finalement, les lésions disparaissent, laissant des plaques d'hypo-pigmentation. L'ensemble de ce processus a pour conséquence l'élimination des cellules portant le chromosome X muté. Les patients atteints d'incontinentia pigmenti peuvent également présenter des défauts dentaires (absence ou déformation de dents), oculaires (anomalies de la vascularisation rétinienne) et plus rarement neurologiques (épilepsie, retard psychomoteur). Une autre caractéristique majeure de ces patients est l'inactivation biaisée du chromosome X observée dans leur compartiment leucocytaire: en effet, alors que l'inactivation du chromosome $\mathrm{X}$ dans chaque cellule est normalement aléatoire, plus de $95 \%$ des leucocytes expriment l'allèle normal. Cela indique que les cellules exprimant le gène muté ont été éliminées, et ceci de façon beaucoup plus précoce qu'au niveau cutané.

La localisation du gène incontientia pigmenti dans la région $\mathrm{Xq} 28$, où se situe NEMO, ainsi que la sensibilité à l'apoptose des rares cellules mutantes dérivées de patients, suggéraient que la voie $\mathrm{NF}-\kappa \mathrm{B}$ puisse être responsable de cette pathologie. De façon remarquable, l'analyse d'une large collection de malades indique que $85 \%$ des cas familiaux ou spontanés d'incontinentia pigmenti présentent un réar- 
rangement identique du locus NEMO. Ce réarrangement fait intervenir des séquences répétées MER67B qui encadrent les exons 4 et 10 . Le résultat final est la production d'un ARNm ne contenant que les trois premiers exons de NEMO et qui code pour une protéine tronquée inactive. Une telle fréquence de réarrangement présente un grand intérêt diagnostic puisqu'un simple test PCR permettra dorénavant d'établir un diagnostic prénatal de la maladie chez plus des trois quarts des familles touchées.

D'autres espoirs concernant cette fois la compréhension de la pathogénie de cette maladie et l'amélioration du sort des patients atteints sont liés à l'analyse, récemment publiée, de lignées murines invalidées pour le gène NEMO localisé aussi, chez la souris, sur le chromosome $\mathrm{X}[8,9]$. Les embryons mâles meurent très tôt $\mathrm{au}$ cours du développement d'une apoptose hépatique massive et l'on observe, quelques heures après la naissance des femelles hétérozygotes, l'apparition d'une dermatose qui va évoluer au cours du temps. L'analyse histologique révèle une très grande similarité entre les différents stades de cette dermatose et ceux qui sont observés chez les malades IP. De plus, les souris $\mathrm{NEMO}^{+/-}$présentent, comme les patients IP, une inactivation biaisée du chromosome $\mathrm{X}$ au niveau des leucocytes. Cet ensemble d'observations indique que les souris invalidées pour le gène NEMO représentent un bon modèle animal pour la pathologie IP. Un tel modèle devrait permettre d'analyser plus précisément les mécanismes moléculaires, encore obscurs, impliqués dans la pathogénie de cette maladie. A un niveau plus fondamental, l'existence d'une pathologie humaine liée à la voie NF- $\kappa B$ devrait apporter de précieuses informations concernant le rôle que joue cet important facteur de transcription in vivo. En tout cas, son rôle au niveau de l'épiderme est maintenant clairement établi.

1. Karin M, Ben-Neriah Y. Phosphorylation meets ubiquitination: The control of NF- $\mathrm{KB}$ activity. Annu Rev Immunol 2000; 18: 621-63.

2. Mercurio F, Zhu H, Murray BW, et al. IKK-1 and IKK-2: cytokine-activated IKB kinases essential for NF-KB activation. Science 1997; 278: 860-6.

3. Yamaoka S, Courtois G, Bessia C, et al. Complementation cloning of NEMO, a component of the

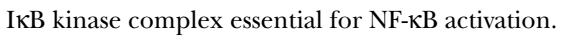
Cell 1998; 93: 1231-40.

4. Seitz CS, Freiberg RA, Hinata K, Khavari PA. $\mathrm{NF}-\mathrm{KB}$ determines localization and features of cell death in epidermis. J Clin Invest 2000; 105 : 25360 .

5. Li Q, Van Antwerp D, Mercurio F, Lee KF, Verma I. Severe liver degeneration in mice lacking the IкB kinase 2 gene. Science 1999; 284 : 321-5.

6. Takeda K, Takeuchi O, Tsujimura T, et al. Limb and skin abnormalities in mice lacking IKK $\alpha$. Science 1999; 284: 313-6.

7. Smahi A, Courtois G, Vabres P, et al. Genomic Rrearrangement in NEMO impairs NF- $\mathrm{BB}$ activation and is a cause of incontinentia pigmenti. Nature 2000 ; 405 : 466-72

8. Makris C, Godfrey VL, Krähn-Senfleben G, et al. Female mice heterozygous for IKK $\gamma /$ NEMO deficiencies develop a dermatopathy similar to the human X-linked disorder Incontinentia Pigmenti. Mol Cell 2000; 5 : 969 -79.

9. Schmidt-Supprian M, Bloch W, Courtois G, et al. NEMO/IKK $\gamma$ - deficient mice model Incontinentia Pigmenti. Mol Cell 2000 ; 5 : 981-92.

\section{Gilles Courtois} Alain Israël

Unité de Biologie moléculaire de l'expression génique, URA 1773 Cnrs, Institut Pasteur, 25, rue du Docteur-Roux, 75724 Paris Cedex 15, France.

\section{口ᄆ BRÈVES}

Un nouveau modèle murin de dystrophie musculaire de Duchenne : la surexpression en cavéoline-3. $\mathrm{La}$ cavéoline 3 est une protéine spécifiquement musculaire localisée au niveau des invaginations membranaires (ou cavéoles) des cellules musculaires. Son absence est responsable d'une forme dominante de myopathie dite des ceintures, encore appelée LGMD1C (limb girdle muscular dystrophy de type 1C). Sa fonction n'est pas encore comprise, de même que son intervention au sein du complexe des nombreuses protéines gravitant autour de la dystrophine. En effet, certains auteurs étaient parvenus à copurifier la cavéoline 3 avec le complexe des protéines associées à la dystrophine (DAP pour dystrophin associated proteins), alors que d'autres ne la considéraient pas comme une DAP $\left(\mathrm{m} / \mathrm{s}\right.$ 1999, $n^{\circ} 2$, p. 279). La seule protéine dont le lien direct avec la cavéoline 3 était démontré est la NO (monoxyde d'azote) synthase neuronale. A défaut de fournir une vue plus éclairée de la physiopathologie de ces dystrophies musculaires, une équipe américano-italienne vient de décrire un nouveau modèle murin de myopathie de Duchenne lié à une surexpression notamment musculaire de la cavéoline 3 [1]. Les souris transgéniques surexprimant cette protéine dans le cerveau, le rein, le foie, le poumon, la rate et le muscle squelettique, présentent un phénotype exclusivement musculaire associant des signes histologiques et biochimiques caractéristiques de nécrose et de régénération à une augmenta- tion des cavéoles sarcolemmiques. De plus, des éléments de fibrose (augmentation importante du tissu conjonctif) sont observés ce qui, paradoxalement, rapproche plus ce modèle de la maladie humaine que de la souris mutante $m d x$, pourtant dépourvue de dystrophine par altération spécifique de son gène. Chez les souris surexprimant la cavéoline 3 , on observe une chute drastique de l'expression de la dystrophine et d'une des protéines qui lui est directement associée, la $\beta$-dystroglycane. Ces résultats suggèrent donc qu'une surexpression de la cavéoline 3 perturbe l'organisation et/ou la mise en place du complexe des DAP.

[1. Galbiati F, et al. Proc Natl Acad Sci USA 2000 ; 97 : 9689-94.] 